(C)2010 IEEE. Personal use of this material is permitted. However, permission to reprint/republish this material for advertising or promotional purposes or for creating new collective works for resale or redistribution to servers or lists, or to reuse any copyrighted component of this work in other works must be obtained from the IEEE. 


\section{Performance improvement with low-cost multi-GNSS receivers}

\author{
Sandra Verhagen \\ Delft Institute of Earth Observation and Space Systems \\ Delft University of Technology \\ Netherlands \\ Email: A.A.Verhagen@TUDelft.nl
}

\author{
Dennis Odijk, Peter J.G. Teunissen and Lennard Huisman \\ GNSS Research Centre \\ Curtin University \\ Perth, Australia \\ Email: D.Odijk@curtin.edu.au
}

\begin{abstract}
In the next five to ten years, the evolution of Global Navigation Satellite Systems (GNSSs) will have a revolutionary impact on the positioning performance. More GNSSs will become available with improved signal characteristics. At the same time, enhancement of receiver technology and algorithms is ongoing. In light of these developments, it is investigated whether highprecision relative positioning with single-frequency receivers will become feasible, and, if so, under which circumstances. We submit that this would open the door to a wide range of applications for instance in the field of mobile Location Based Services for which users do not have professional receivers at their disposal.

The closed form expression of the single-frequency Ambiguity Dilution of Precision (ADOP) gives a clear insight into how and to what extent the various factors of the underlying singlefrequency model contribute to the overall ambiguity resolution performance. Furthermore, numerical studies indicate that for benign dynamics single-frequency RTK becomes feasible for baselines up to about $\mathbf{1 0 - 1 5} \mathbf{~ k m}$ if GPS+Galileo is used. In this contribution we will present analytical and numerical results. Ambiguity resolution performance as function of number of epochs, receiver noise and baseline length will be analyzed, and compared for ideal circumstances as well as for situations with bad satellite visibility and/or multipath. Furthermore, different next generation GNSS configurations will be considered.

Based on the results, it is predicted that for rapid, short baseline $\mathrm{cm}$-level positioning, low-cost single-frequency receivers will become very competitive in comparison to their more expensive dual-frequency cousins.
\end{abstract}

\section{INTRODUCTION}

In the next five to ten years, the evolution of Global Navigation Satellite Systems (GNSSs) will have a revolutionary impact on the positioning performance. Until recently, GNSS users have been deeply dependent on the American Global Positioning System (GPS), since it was the only fully operational system with more than 28 satellites in orbit. However, the Russian Glonass is approaching full operational capability; Galileo should be available in 2020; and the Chinese Compass is planned to be globally available in 2015 . That will bring the total number of GNSS satellites to over 100. Besides the increased number of satellites, technological advancements will induce a dramatic performance improvement in the near future. The specific case that informs this paper is the performance improvement due to the availability of more GNSSs.

State-of-the-art professional receivers are already capable of receiving signals from GPS, GLONASS, and Galileo. The reason for this stems from the idea that more ranging signals will enhance the accuracy, availability, and integrity. Although this is true, the performance improvement of a three- or foursystem receiver is modest compared to a two-system receiver. Still, it is a desire of professional GNSS users to be able to use all available signals, that is GNSSs. This desire can be easily satisfied since professional receivers are already very complex and expensive; to be able to track more signals only has a relatively small impact on hardware and cost of the receiver.

It is important to note that tracking the signals of multiple GNSSs can be achieved without high demands on the receiver architecture, and thus the costs, as long as the GNSSs transmit on the same frequencies. This fact has motivated us to investigate whether it is desirable for low-cost receivers, such as receivers in car navigation systems, personal digital assistants, and mobile phones, to be able to track the signals of multiple GNSSs. Low-cost receivers are often singlefrequency receivers, targeted to provide meter-level positioning accuracies. Early tests indicated that the performance of lowcost single-frequency receivers will improve dramatically if they are capable of tracking more than one GNSS.

Presently, low-cost single-frequency RTK systems lack real-time performance due to the weaknesses of the singlefrequency GPS-only model, see e.g. [1], [6], [8], [11], [17]. The objective is to investigate whether high-precision relative positioning with single-frequency receivers will be feasible, and, if so, under which circumstances. We submit that this would open the door to a wide range of applications for instance in the field of mobile Location Based Services, precision agriculture, mining, deformation monitoring, surveying and mapping, e.g. [3], [9], [14], [16], [26], for which users do not have professional receivers at their disposal.

This contribution starts with an analysis based on the closed form expression of the single-frequency Ambiguity Dilution of Precision (ADOP). This gives a clear insight into how and to what extent the various factors of the underlying single-frequency model contribute to the overall ambiguity resolution (AR) performance. Next, the ambiguity resolution performance as function of number of epochs, receiver noise and baseline length will be analyzed based on simulation studies, both for ideal circumstances as well as for situations with multipath. 


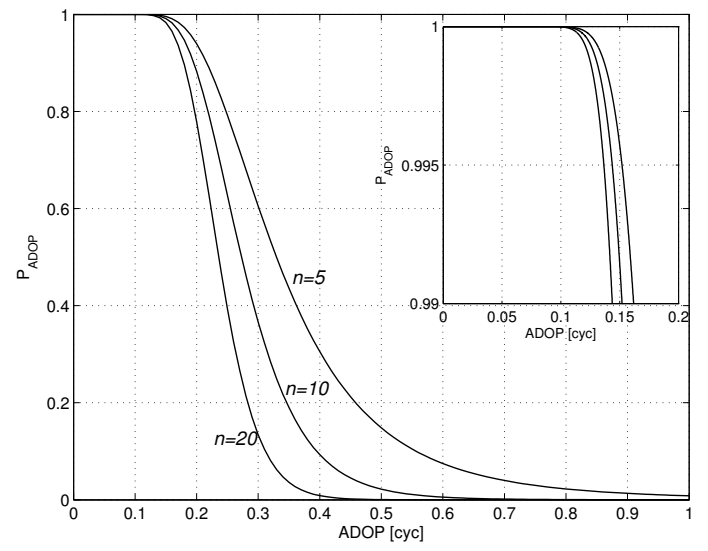

Fig. 1. $P_{\mathrm{ADOP}}$ as function of ADOP.

\section{SingLE-FREQUENCY ADOP}

The key to rapid and high-precision GNSS positioning is the use of carrier-phase observations, which have mm-level precision while code observations only have a precision at the dm-level. In order to exploit the very precise carrier-phase measurements, first the unknown integer number of cycles of the observed carrier phase has to be resolved. The linearized double-difference GNSS model can be written as:

$$
y=B b+A a+e, \quad b \in \mathbb{R}^{v}, a \in \mathbb{Z}^{n}
$$

where $y$ is the vector with double-differenced code and phase observables; $b$ is the $v$-vector with unknown real-valued parameters, such as the baseline increments, ionosphere and troposphere parameters; $a$ is the $n$-vector with the unknown integer ambiguities; $e$ is the noise vector. The matrices $B$ and $A$ link the unknown parameters to the observables. It is generally assumed that $y$ follows the normal distribution, with zero-mean noise and the associated variance matrix $Q_{y y}$ captures the measurement precision.

Solving model (1) in a least-squares sense provides the so-called float solution, where the integer constraint on the carrier-phase ambiguities, i.e. $a \in \mathbb{Z}^{n}$, is not considered. This is done in a second step, the ambiguity resolution (AR) step, based on the float ambiguities $\hat{a}$ and associated variance matrix $Q_{\hat{a} \hat{a}}$. The integer least-squares (ILS) estimator is proven to be optimal in the sense that it maximizes the probability of correct integer estimation, [22]. A well-known and efficient implementation of the ILS-principle is the LAMBDA method, [18]. After resolving the integer ambiguities $\breve{a}$, the final step is to adjust the float solution of $b$ conditioned on the fixed integer solution. This provides the fixed baseline solution $\breve{b}$.

Correct integer estimation is essential to guarantee that $\breve{b}$ will have $\mathrm{cm}$-level precision. Hence, the probability of correct integer estimation, called success rate, is a valuable measure to assess the positioning performance. Unfortunately, no analytical expression is available to compute the ILS success rate exactly. Several approximations were proposed in the past, see [24]. In this contribution empirical success rates based on Monte Carlo simulations will be used.
In [19] the Ambiguity Dilution of Precision (ADOP) was introduced as an AR performance measure. It is defined as:

$$
\mathrm{ADOP}=\sqrt{\left|Q_{\hat{a} \hat{a}}\right|^{\frac{1}{n}}}
$$

The ADOP measure has the unit of cycles, and it is invariant to the decorrelating $Z$-transformation of the LAMBDA method. It is equal to the geometric mean of the standard deviations of the ambiguities if these would be completely decorrelated. Hence, the ADOP approximates the average precision of the transformed ambiguities. The ADOP can also be used to get an approximation of the ILS success rate:

$$
P(\check{a}=a) \approx P_{\mathrm{ADOP}}=\left(2 \Phi\left(\frac{1}{2 \mathrm{ADOP}}\right)-1\right)^{n}
$$

Fig. 1 shows the relation between ADOP and $P_{\mathrm{ADOP}}$ for different values of $n$. From this figure it can be concluded that for successful ambiguity resolution the ADOP should be smaller than 0.15 cycles.

It is possible to derive closed-form expressions for ADOP. In [13] this was done for a hierarchy of multi-frequency single-baseline GNSS models. The closed-form expressions give a clear insight into how and to what extent the various factors of the underlying GNSS model contribute to the overall AR performance, see [12]. The closed-form expression for the ADOP of the single-frequency model corresponding to a moving receiver covering a short time span (no change in satellite geometry) can be derived as (see table 8 in [13], use $j=1)$ :

$$
\mathrm{ADOP}=f_{c} \times f_{s} \times f_{g} \times f_{b}
$$

with

$$
\begin{aligned}
& f_{c}=\frac{\sigma_{\phi}}{\lambda} \\
& f_{s}=\left[2 \sigma_{t}^{2}\right]^{\frac{1}{2}} \\
& f_{g}=\left[\frac{\sum_{s=1}^{m} w_{s}}{\prod_{s=1}^{m} w_{s}}\right]^{\frac{1}{2(m-1)}} \\
& f_{b}=\left[1+\eta \cdot \frac{\kappa}{1+\kappa}\right]^{\frac{1}{2}}\left[1+\eta \cdot \frac{(2 \kappa+1)^{2}}{1+\kappa(1+\eta)}\right]^{\frac{v}{2(m-1)}}
\end{aligned}
$$

and:

$\sigma_{\phi}$ undifferenced phase standard deviation [m]

$\lambda$ carrier wavelength $[\mathrm{m}]$

$\sigma_{t}^{2}$ variance scale factor

$m$ number of satellites

$w_{s}$ elevation dependent weights, $s=1, \ldots, m$

$\eta=\frac{\sigma_{p}^{2}}{\sigma_{\phi}^{2}}$ and $\kappa=\frac{\sigma_{1}^{2}}{\sigma_{p}^{2}}$

$\sigma_{p}$ undifferenced code standard deviation [m]

$\sigma_{\iota}$ undifferenced standard deviation of ionosphere observables [m]

In Eq.(4) four factors are distinguished, which will be discussed and analyzed in more detail below.

\section{Carrier frequency}

The first factor in Eq.(4), $f_{c}$, depends on the wavelength of the carrier and the undifferenced standard deviation of the 


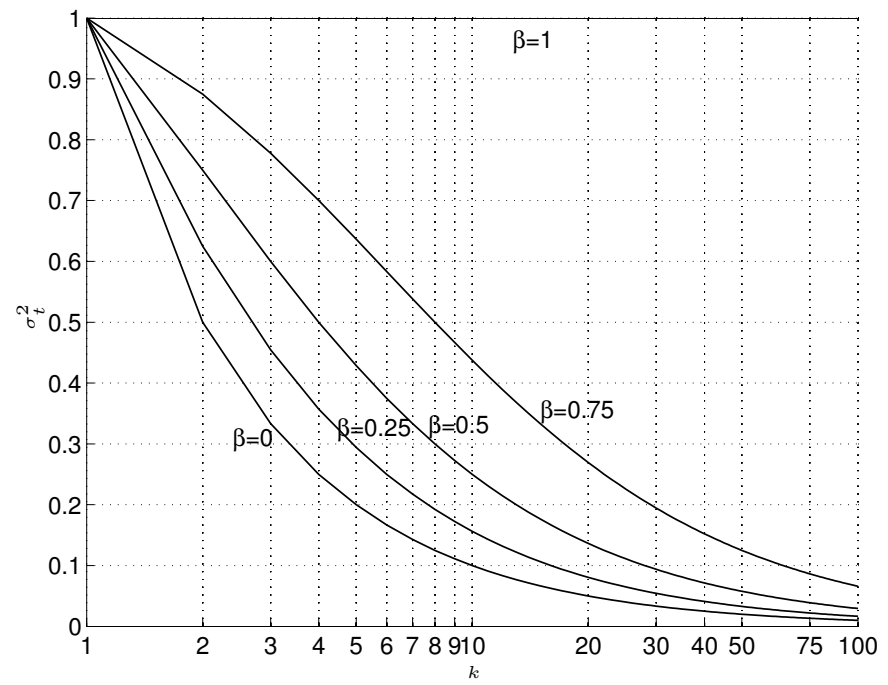

Fig. 2. Relation between the variance scale factor $\sigma_{t}^{2}$ and number of epochs $k$ for various time correlations $\beta$.

corresponding carrier phase observable in zenith direction. Here, it is especially interesting to look into the effect of the wavelength, since the scale factor in the second term may account for different precisions. It follows that if all other factors would be the same, the ADOP with L5 would be $\frac{\lambda_{\mathrm{L} 1}}{\lambda_{\mathrm{L} 5}}=0.75$ smaller than the ADOP with L1. This is purely the effect of the longer wavelength.

\section{Scale factor}

The second term in Eq.(4), $f_{s}$, depends on the variance scale factor $\sigma_{t}^{2}$. If $\sigma_{t}^{2}<1$ this can be either due to enhanced measurement precision, or due to an increased number of epochs $k$. In the first case it is assumed that the variance of code and phase observations is improved with the same factor $\sigma_{t}^{2}$. In the second case the scale factor would be equal to:

$$
\sigma_{t}^{2}=\frac{1+\beta}{k-(k-2) \beta}
$$

where $\beta(0 \leq \beta<1)$ describes the correlation parameter of a first-order autoregressive time process. Hence, $\beta=0$ means that time correlation is absent and $\sigma_{t}^{2}=\frac{1}{k}$, while $\beta=1$ would mean that the observations are fully correlated between the epochs and $\sigma_{t}^{2}=1$. Fig. 2 shows the relation between the variance scale factor $\sigma_{t}^{2}$ and the number of epochs $k$ for various time correlations $\beta$.

\section{Geometry factor}

The factor $f_{g}$ in Eq.(4) depends on the satellite geometry, assuming that an elevation dependent weighting scheme is applied, such that degraded measurement precision at lower elevations due to a longer signal travel path through the atmosphere and potential multipath effects is taken into account. Without elevation dependent weighting $f_{g}=1$.

The elevation dependent weight function applied here is:

$$
w_{s}=\left(1+10 \exp \left\{-\frac{e_{s}}{10}\right\}\right)^{-2}
$$

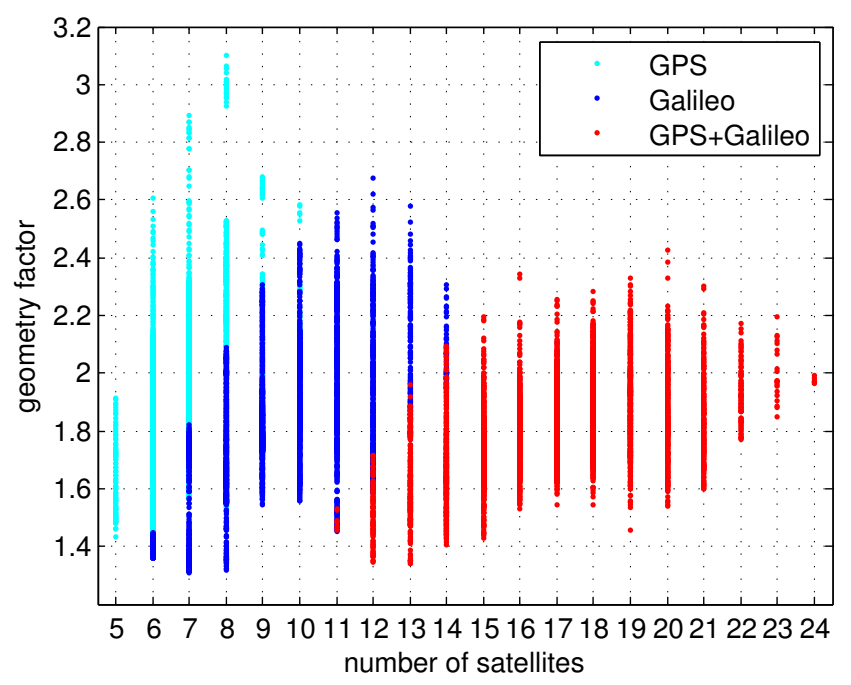

Fig. 3. Geometry factor $f_{g}$ for 10-day period as function of number of satellites, for an equatorial, mid-latitude and high-latitude location.

where $e_{s}$ is the elevation [deg] of satellite $s$.

Fig. 3 shows the geometry factor $f_{g}$ for a 10-day period (covering a full repeat orbit period of Galileo) for GPS, Galileo and GPS+Galileo, using Eq.(7). Each dot represents the number of satellites and corresponding $f_{g}$ for one time instant during the 10-day period at one of the geographical latitudes.

\section{Baseline factor}

The last term in Eq.(4), $f_{b}$, depends on different factors. Firstly, it depends on the variance ratio of the code and phase observables. In this contribution it is assumed that the code standard deviation is 100 times higher than the phase standard deviation, and hence $\eta=10^{4}$.

Furthermore, $f_{b}$ depends on the variance ratio of the ionosphere and code observables. This means that the underlying model is the ionosphere-weighted model, see e.g. [10], in which either a priori information on the ionosphere delays is used in the form of ionosphere observables (if no external information is available, the observables are set to zero). The uncertainty of these observables must be taken into account; it is modeled with a standard deviation $\sigma_{\iota}$ depending on the baseline length. If the baseline is sufficiently short, the double difference ionosphere observables will become zero, and $\sigma_{\iota}$ is set to zero.

As a rule-of-thumb here a relation of $4 \mathrm{~mm} / 10 \mathrm{~km}$ is used, i.e. $\sigma_{\iota}=4 \mathrm{~mm}$ for a $10 \mathrm{~km}$ baseline. Furthermore, the same elevation dependent weighting function is applied as to the code and phase observables, implying that $\sigma_{\iota}$ is the standard deviation in zenith direction.

Finally, $f_{b}$ depends on the number of visible satellites $m$ and the number of unknown 'baseline' parameters $v$, which may include troposphere and ionosphere delays next to the three baseline unknowns. Here it is assumed that zenith troposphere delays are to be estimated for baseline lengths longer than 10 

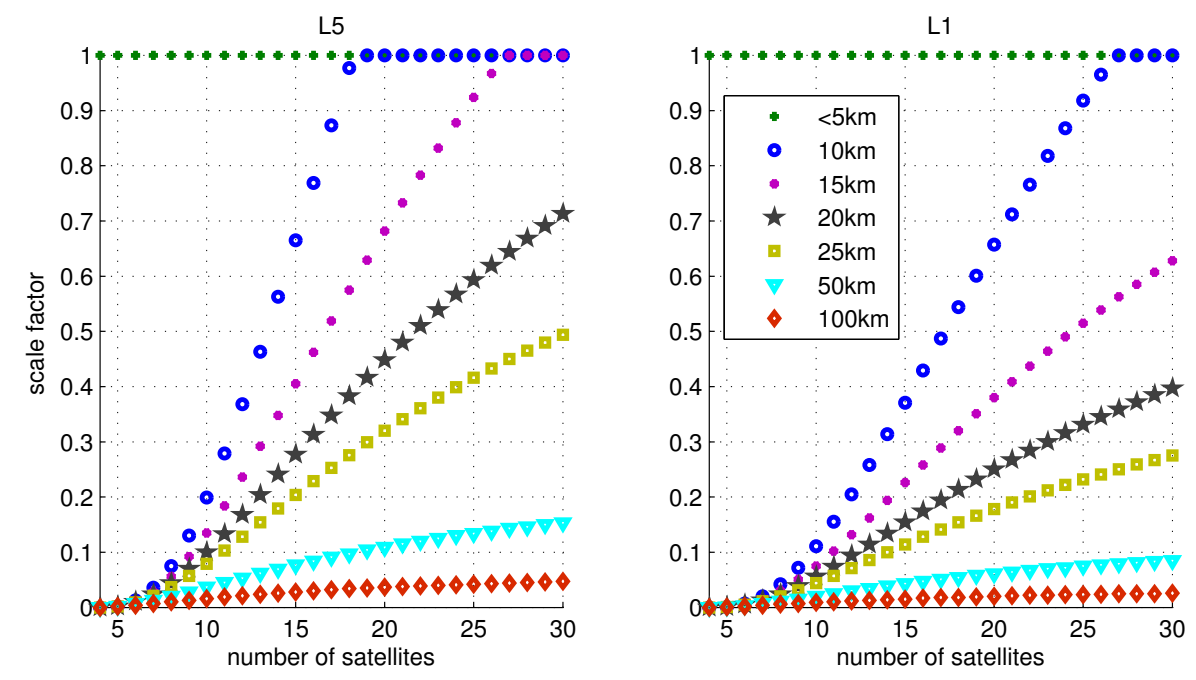

Fig. 4. Required scale factor as function of number of satellites in order to obtain ADOP $<0.15$ cycles. Geometry factor is equal to 2.5 . L5 (left) and L1 (right) frequency.
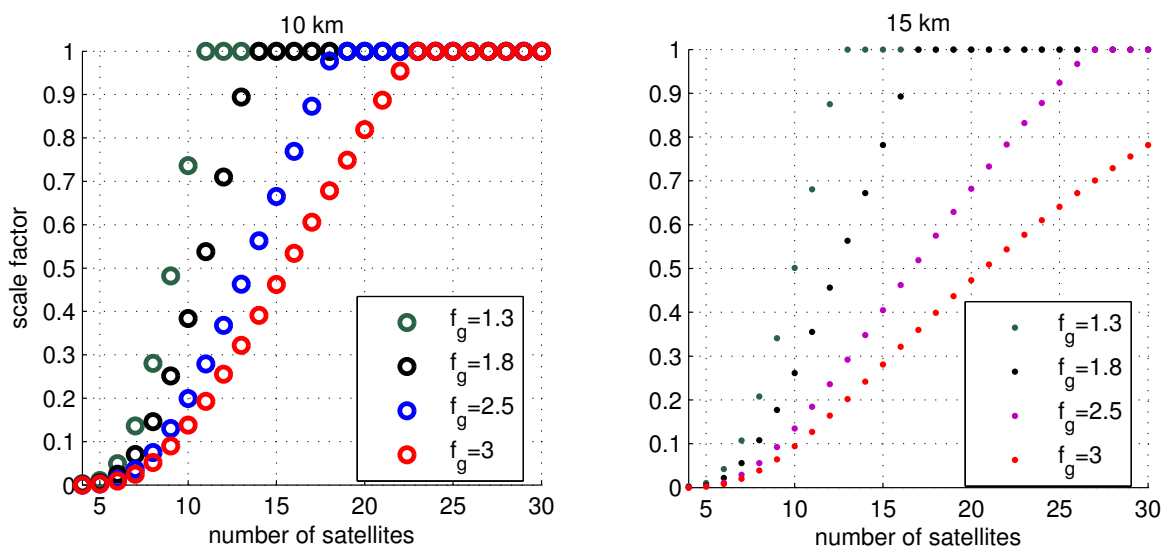

Fig. 5. Required scale factor as function of number of satellites in order to obtain ADOP $<0.15$ cycles for different geometry factors. $10 \mathrm{~km}$ (left) and 15 $\mathrm{km}$ (right) baseline.

$\mathrm{km}$, so that $v=4$, while it is assumed that the troposphere can be neglected in the double differenced model for shorter baselines, in which case $v=3$.

\section{Analysis of factors contributing to $A D O P$}

Fig. 4 shows how the different factors in Eq.(4) contribute to the ADOP, except for the geometry factor, which is fixed to a value of $f_{g}=2.5$, which is a conservative choice as can be seen from Fig. 3 .

Main conclusions from Fig. 4 are that especially for shorter baselines with increasing number of satellites, the required scale factor may be much larger. Hence, it can be expected that fast ambiguity resolution becomes feasible with GPS+Galileo. Furthermore, with increasing baseline length, a very small scale factor is required to obtain a sufficiently small ADOP. See Fig. 2 for the relation between the scale factor and number of epochs required, assuming the precision cannot be improved otherwise.

Fig. 5 shows again the required scale factor to obtain an ADOP of less than 0.15 cycles, but now for different geometry factors, and only for baselines of 10 and $15 \mathrm{kms}$, since Fig. 4 indicates that for longer baselines fast ambiguity resolution will not be feasible. According to Fig. 3 the factor $f_{g}$ will generally be smaller than 2.5 , and on average it will be around 1.8 (both for Galileo and GPS+Galileo). For this latter value, the figure shows that with more than 10 visible satellites the ADOP will be below 0.15 cycles even with a scale factor larger than 0.5 . With less visible satellites, as will often be the case with GPS- or Galileo-only, the scale factor must be much smaller, implying that many more epochs and/or higher precision is required.

\section{AMBIGUITY RESOLUTION PERFORMANCE}

In Section II the influence of different factors on AR performance has been analyzed. A preliminary conclusion from this analysis was that single-frequency RTK with combined GPS+Galileo will be feasible with baselines shorter than 15 $\mathrm{km}$. It will now be investigated whether this is indeed a viable conclusion by assessing the AR performance based on 


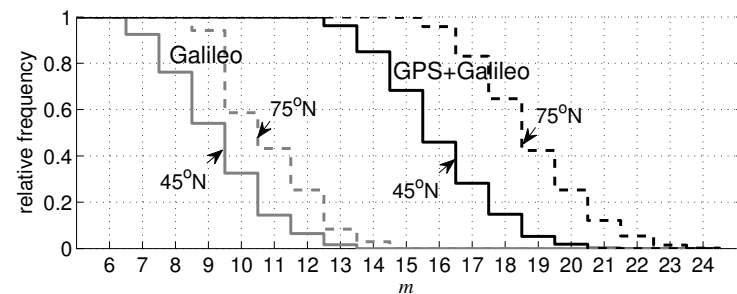

Fig. 6. Relative frequencies that more than $m$ satellites are visible for 10-day period.

simulation studies. In this case, the performance measure that will be used is the AR success rate.

First the AR performance of Galileo versus GPS+Galileo will be compared, assuming clear sky conditions. This will give a good impression of what can be achieved for applications like farming and vehicle navigation (road, rail, river). Another important application field of low-cost, singlefrequency RTK would be Location Based Services and Tracking and Tracing in urban areas. Those areas are known to be problematic in terms of satellite visibility and multipath. Therefore, also simulation studies have been carried out to show the improved AR performance with GPS+Galileo in those conditions.

\section{A. Clear sky conditions}

In order to analyze the AR performance of Galileo and GPS+Galileo, the future Galileo constellation of 30 satellites and the nominal GPS the constellation of 24 satellites were considered. Two geographical locations were chosen: $45^{\circ} \mathrm{N}$ and $75^{\circ} \mathrm{N}$. The mid-latitude location was selected because here on average the least number of satellites are visible, while at the higher latitude the opposite is true, see Fig. 6. Furthermore, the satellite geometry is generally worse at $45^{\circ} \mathrm{N}$, because no satellites from Northern directions are visible (azimuths between 330 and $30^{\circ}$ ).

The following settings were applied:

$$
\begin{aligned}
\sigma_{\phi} & =2 \mathrm{~mm}, \sigma_{p}=20 \mathrm{~cm} \\
\sigma_{\iota} & =2,4,6,8 \mathrm{~mm} \\
\lambda & =25.48 \mathrm{~cm}(\mathrm{~L} 5 \text { frequency) } \\
w_{s} & =\left(1+10 \exp \left(-e_{s} / 10\right)\right)^{-2} \\
v & =3 \text { or } 4 \text { (troposphere float and fixed, respectively) }
\end{aligned}
$$

A mask angle of $10^{\circ}$ was used. Applying the rule-of-thumb relation between $\sigma_{\iota}$ and the baseline length, this corresponds to baselines of 5, 10, 15 and $20 \mathrm{~km}$.

The standard deviations of the code and phase observations are relatively conservative compared to the expected thermal noise characteristics of the future GNSS signals as presented in [15]. Here we choose somewhat higher standard deviations to account for multipath and other residual effects, as well as to simulate the performance with low-grade receivers. Because of the elevation dependent weighting, the stochastic model may in this way be assumed to account for residual troposphere delays for baselines of around $10 \mathrm{~km}$. For completeness,
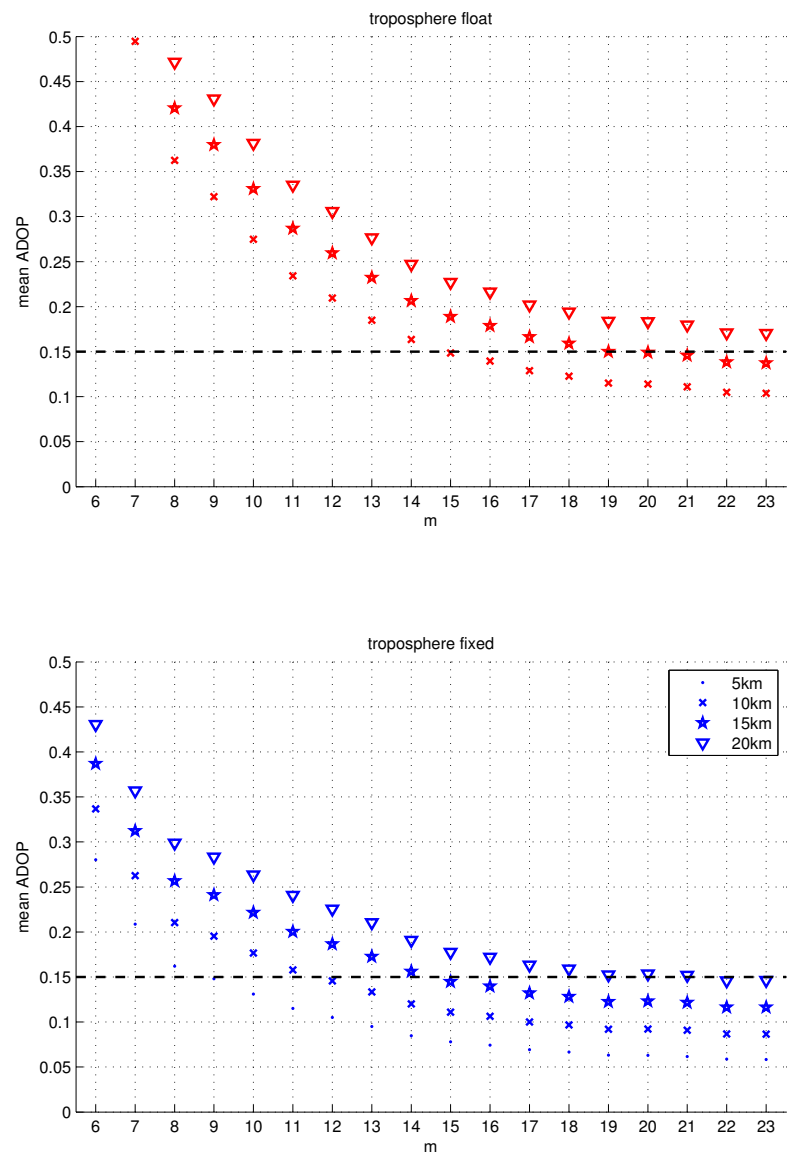

Fig. 7. Single-epoch mean ADOPs [cycles] as function of number of satellites. Top: troposphere float. Bottom: troposphere fixed.

however, both the troposphere fixed and float model were applied for baselines of $10 \mathrm{~km}$ and longer.

In order to get a first impression of the potential AR performance with single-frequency RTK, the ADOPs for Galileo and GPS+Galileo were determined for a 10-day period. Fig. 7 presents the mean ADOPs as function of the number of satellites with $\sigma_{t}^{2}=1$ (i.e. the mean for each $m$ is calculated over all instances that $m$ number of satellites are visible during the 10-day period). By taking the mean ADOPs, it turned out that the impact of the geometry factor $f_{g}$ is averaged out, and therefore the mean ADOPs for the two latitudes are approximately equal. The values in Fig. 7 are in fact the average values for the two locations.

It becomes clear that if the troposphere float model is to be used, single-epoch AR with these settings is not feasible, unless more than 15 and 19 satellites are visible for a 10 and $15 \mathrm{~km}$ baseline, respectively. Only then the mean ADOP is below 0.15 cycles. If, on the other hand, the troposphere fixed model can be applied, instantaneous AR seems feasible for the 10 and $15 \mathrm{~km}$ baselines on the condition that more than 12 and 15 satellites, respectively, are visible. With combined GPS-Galileo this will be the case for a large percentage of time, see Fig. 6. For very short baselines, Galileo-only may already suffice. 
In order to get a better impression of the AR performance, Fig. 8 shows the percentage of time that the AR success rate is above 0.99 as a function of the variance scale factor $\sigma_{t}^{2}$. Results are presented separately for the troposphere fixed and float models, and for the two latitudes.

For the troposphere float case (top panels) it is still possible to obtain success rates above 0.99 with GPS+Galileo (red markers) for a baseline of $10 \mathrm{~km}$ for most of the time. However, fast AR with a $15 \mathrm{~km}$ baseline will require of very small variance scale factor. With Galileo-only (blue markers), already with a $10 \mathrm{~km}$ baseline the AR performance is very weak.

For the troposphere fixed case (bottom panels) it follows that fast AR should be possible most of the time, even with baselines of $15 \mathrm{~km}$ if GPS+Galileo can be used. With Galileoonly, high success rates can only be obtained for very short baselines or small variance scale factors (corresponding to more than 10-20 epochs in the absence of time correlation).

\section{B. Degraded satellite visibility}

In order to study the performance in degraded satellite visibility conditions, the same simulation settings as in Section III-A were applied, but now it was assumed that all satellites with an azimuth between $0^{\circ}$ and $70^{\circ}$ and an elevation smaller than $50^{\circ}$ were blocked. As a result $1-4$ Galileo satellites were blocked, with an average of 2-3 satellites for the complete 10day period. For combined GPS+Galileo, 1-7 satellites were blocked, with an average of 3-4. Fig. 9 is similar to Fig. 8, but now only the results for a $10 \mathrm{~km}$ baseline are shown, with and without satellite masking. As expected, the percentage of time that a high success rate can be obtained becomes much lower, especially for Galileo-only. On the other hand, with GPS+Galileo, the percentage of time is still reasonably high even for scale factors close to 1 , especially if the troposphere fixed model (bottom panels) can be applied. Hence, even though more epochs of data would be required, it seems that fast ambiguity resolution (AR) is still feasible in degraded satellite visibility conditions for $10 \mathrm{~km}$ baselines. In more severe circumstances, it might be necessary to add a third GNSS, e.g. Glonass.

\section{Multipath conditions}

In order to show the improved AR performance with GPS+Galileo for single-frequency RTK in multipath conditions a simulation study has been carried out for which one day of GPS and Galileo data was simulated for a $10 \mathrm{~km}$ baseline at a mid-latitude location $\left(50^{\circ} \mathrm{N}\right)$. In this case, the GPS constellation as of December 2005 (29 satellites in orbit) was used. The undifferenced code standard deviation was set to $1 \mathrm{~m}$ both for GPS and Galileo. The undifferenced phase standard deviations for L1 and L5 were set to 1 and $1.3 \mathrm{~mm}$, respectively, again both for GPS and Galileo.

Multipath was simulated assuming a reflective surface at 50 meters distance from the rover receiver, causing the multipath biases to be depending on the signal attenuation, see [5].
TABLE I

Percentage of time AmBIguities fixed and correct (SUCCESS) OR fixed and wrong (FAILURE).

\begin{tabular}{|c|ll|ll|}
\hline baseline & \multicolumn{2}{|c|}{ GPS } & \multicolumn{2}{c|}{ GPS+Galileo } \\
& L1 & L5 & L1 & L5 \\
\hline success & 0.8 & 5.7 & 98.3 & 99.95 \\
\hline failure & 0 & 0 & 0.8 & 0.05 \\
\hline
\end{tabular}

Table I presents the AR performance characteristics based on single-epoch ambiguity resolution with Fixed-Failure Rate Ratio Test, see [23], using a fixed failure rate of $0.1 \%$. The table shows the percentage of time that the ambiguities were accepted and correct, as well as that they were accepted but wrong.

With GPS-only the fixing rate is very low. With combined GPS+Galileo, a dramatic performance improvement can be observed: successful instantaneous ambiguity resolution is possible nearly all day. Obviously, due to the larger number of visible satellites, the propagation of the multipath biases is much less compared to the GPS-only case.

\section{CONCLUSION}

Single-frequency RTK with a single GNSS is only feasible with very short baselines $(<5 \mathrm{~km})$, and even then at some locations more than 10-15 epochs would be required to obtain sufficiently high success rates.

A dual-constellation GNSS will enhance the ambiguity resolution performance of single-frequency RTK dramatically. Instantaneous success rates above 0.99 are obtained with baselines up to $15 \mathrm{~km}$ in clear sky conditions. This result is based on a conservative choice for the stochastic model, for which it can be assumed that residual troposphere effects are within the noise level (i.e. troposphere fixed model can be used). In severe circumstances, where it would be more appropriate to use the troposphere float model, fast and reliable AR will still be possible with GPS+Galileo for baselines up to $10 \mathrm{~km}$.

In situations with poor satellite visibility and/or multipath, the AR performance may be severely degraded. Results in this contribution, however, show that the performance with GPS+Galileo may still suffice, and is by far not degraded as much as with a single GNSS.

\section{ACKNOWLEDGMENT}

The research of S. Verhagen is supported by the Dutch Technology Foundation STW, applied science division of NWO and the Technology Program of the Ministry of Economic Affairs.

Professor P.J.G. Teunissen is the recipient of an Australian Research Council Federation Fellowship (project number FF0883188): this support is greatly acknowledged. 

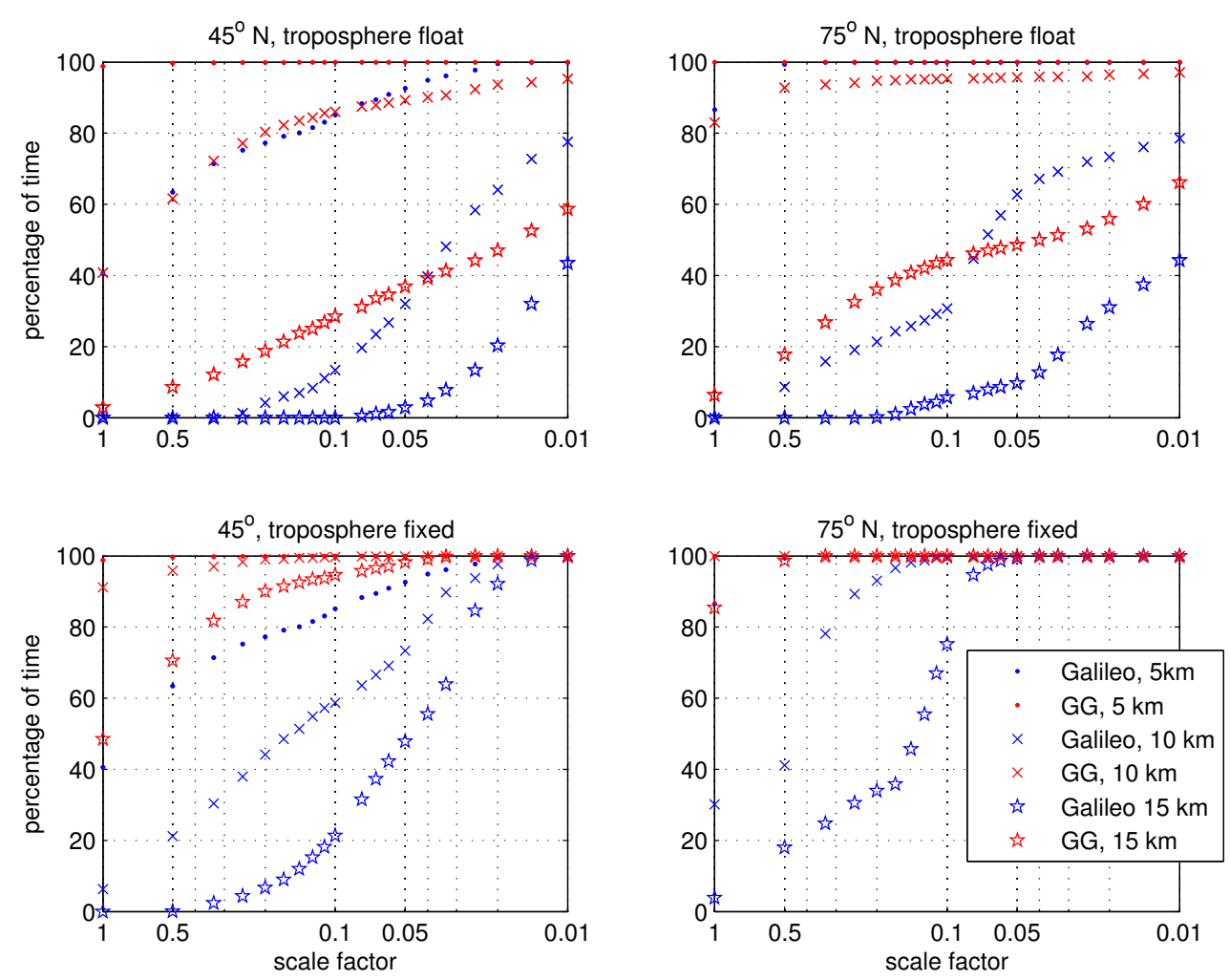

Fig. 8. Percentage of time that success rate is above 0.99 as a function of the scale factor $\sigma_{t}^{2}$. Top: troposphere float. Bottom: troposphere fixed. Left: $45^{\circ} \mathrm{N}$. Right: $75^{\circ} \mathrm{N}$.
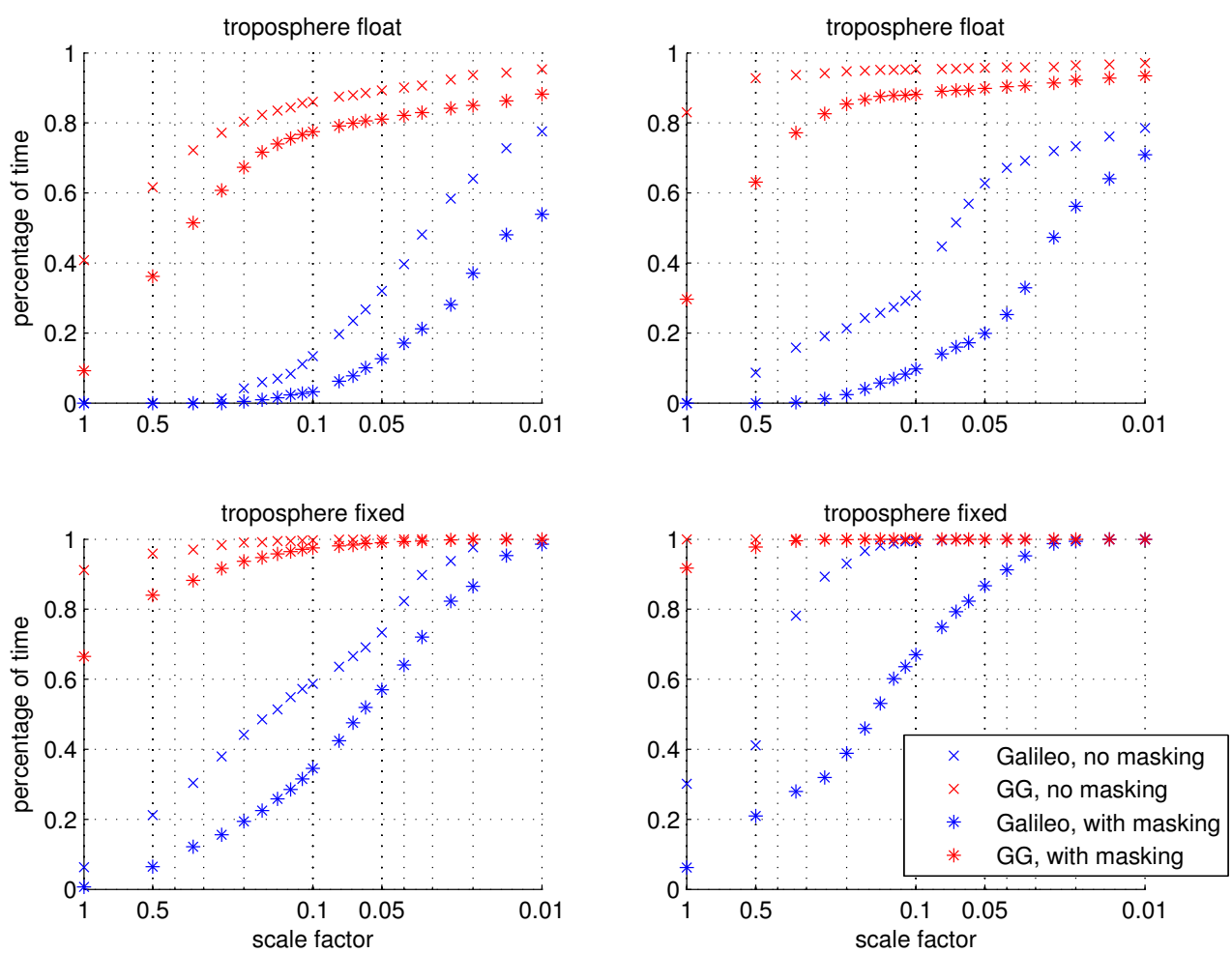

Fig. 9. Percentage of time that success rate is above 0.99 as a function of the scale factor $\sigma_{t}^{2}$ in clear sky and degraded satellite visibility conditions. Top: troposphere float. Bottom: troposphere fixed. Left: $45^{\circ} \mathrm{N}$. Right: $75^{\circ} \mathrm{N}$ 


\section{REFERENCES}

[1] Beran, T., Bisnath, S.B., and Langley, R.B. (2004). Evaluation of HighPrecision, Single-Frequency GPS Point Positioning Models. Proc. of the 17th International Technical Meeting of the Satellite Division of The Institute of Navigation (ION GNSS 2004), Long Beach, CA, September 2004, pp. 1893-1901.

[2] Blewitt, G. (1989). Carrier-phase ambiguity resolution for the Global Positioning System applied to baselines up to $2000 \mathrm{~km}$. Journal of Geophysical Research, 94(B8), 10187-10302.

[3] Denham, J.L., Whitlock, A.K., and Yule, D. (2006). Contemporary Position and Navigation Needs of Precision Agriculture and Victorias GPSnet CORS Network, Proc. of IGNSS Symposium, Holiday Inn Surfers Paradise, Australia.

[4] Dong, D. and Bock, Y. (1989). Global Positioning System network analysis with phase ambiguity resolution applied to crustal deformation studies in California. Journal of Geophysical Research, 94(B4), 39493966.

[5] Huisman, L., Teunissen, P.J.G., and Odijk, D. (2010). On the Robustness of Next Generation GNSS Phase-only Real-Time Kinematic Positioning, Proc. of FIG Congress, Sydney, Australia, April 2010.

[6] Johnson, L., and Van Diggelen, F. (1998). Advantages of a Combined GPS+GLONASS Precision Sensor for Machine Control Applications in Open Pit Mining. Proc. of IEEE PLANS 1998, Palm Springs, CA, April 1998, pp.549-454.

[7] Kim, D. and Langley, R. B. (2000). GPS ambiguity resolution and validation: methodologies, trends and issues. Proc. of 7th GNSS Workshop, Seoul, Korea.

[8] Milbert, D. (2005). Influence of pseudorange accuracy on phase ambiguity resolution in variousGPSmodernization scenarios. Navigation, 52(1), 2938 .

[9] Millner, J., Hale, M., Standen, P., and Talbot, N. (2004). The development and enhancement of a GNSS/GPS infrastructure to support Location Based Service Positioning Systems in Victoria, Proc. of International Symposium on GNSS/GPS, Sydney, Australia.

[10] Odijk, D. (2002). Fast precise GPS positioning in the presence of ionospheric delays. Ph.D. thesis, Publications on Geodesy, 52, Netherlands Geodetic Commission, Delft.

[11] Odijk, D., Traugott, J., Sachs, G., Montenbruck, O., and Tiberius, C. (2007). Two Approaches to Precise Kinematic GPS Positioning with Miniaturized L1 Receivers, Proc. of ION GNSS 2007, Fort Worth TX, pp.827-838.

[12] Odijk, D. and Teunissen, P. J. G. (2007). Sensitivity of ADOP to changes in the single-baseline GNSS model. Artificial Satellites, 42(2), 71-96.

[13] Odijk, D. and Teunissen, P. J. G. (2008). ADOP in closed form for a hierarchy of multi-frequency single-baseline GNSS models. Journal of Geodesy, 82(8), 473-492.

[14] Saeki, M. and Hori, M. (2006). Positioning System Using Low-Cost L1 GPS Receivers, Computer-Aided Civil and Infrastructure Engineering, 21(4), 258-267.

[15] Simsky, A., Sleewaegen, J. M., Hollreiser, M., and Crisci, M. (2006). Performance Assessment of Galileo Ranging Signals Transmitted by GSTB-V2 Satellites. Proc. of ION GNSS 2006, Fort Worth TX.

[16] Squarzonia, C., Delacourta, C., and Allemanda, P. (2005). Differential single-frequency GPS monitoring of the La Valette landslide (French Alps). Engineering Geology, 79, 215-229.

[17] Takasu, T. and Yasuda, A. (2008). Evaluation of RTK-GPS Performance with Low-cost Single-frequency GPS Receivers, Proc. of International Symposium on GPS/GNSS 2008 Tokyo, Japan, pp. 852-861.

[18] Teunissen, P. J. G. (1993). Least squares estimation of the integer GPS ambiguities. Invited lecture, Section IV Theory and Methodology, IAG General Meeting,Beijing.

[19] Teunissen, P. J. G. (1997). A canonical theory for short GPS baselines. Part IV: Precision versus reliability. Journal of Geodesy, 71, 513-525.

[20] Teunissen, P. J. G. (1998a). GPS carrier phase ambiguity fixing concepts. In: PJG Teunissen and Kleusberg A, GPS for Geodesy, SpringerVerlag,Berlin.

[21] Teunissen, P. J. G. (1998b). Success probability of integer GPS ambiguity rounding and bootstrapping. Journal of Geodesy, 72, 606-612.

[22] Teunissen, P. J. G. (1999). An optimality property of the integer leastsquares estimator. Journal of Geodesy, 73(11), 587-593.

[23] Teunissen P.J.G., and Verhagen, S. (2009). The GNSS ambiguity ratiotest revisited: A better way of using it. Survey Review, 41(312), 138-151
[24] Verhagen, S. (2005). On the reliability of integer ambiguity resolution. Navigation, 52(2), 99-110.

[25] Verhagen, S. and Joosten, P. (2004). Analysis of integer ambiguity resolution algorithms. European Journal of Navigation, 2(4), 38-50.

[26] Wirola, L., Alanen, K., Kappi, J., and Syrjarinne, J. (2006). Bringing RTK to Cellular Terminals Using a Low-Cost Single-Frequency AGPS Receiver and Inertial Sensors. Proceedings IEEE/ION PLANS 2006, San Diego CA, pages 645-652. 